\title{
Développement du piétin-verse dû à Pseudocercosporella herpotrichoides sur des associations variétales de blé tendre d'hiver
}

\author{
L Saur $^{1}$, B Mille ${ }^{2 *}$ \\ 'SRIV, Inra, BP 29, F-35650 Le Rheu; \\ ${ }^{2} S R I V$, Inra, route de Saint-Cyr, F-78026 Versailles cedex, France
}

(Reçu le 19 octobre 1995 ; accepté le 8 janvier 1997)

\begin{abstract}
Summary - Disease progress of Pseudocercosporella herpotrichoides in mixed stands of winter wheat cultivars. Epidemic development of Pseudocercosporella herpotrichoides in pure stands of a susceptible variety and a variety with the $P c h l$ resistance gene, and in their (1:1) mixture was examined in six situations differing for location, fungicide protection or contamination. At growth stade 32, no significant difference was found between the disease level in mixtures and the mean of the disease levels of the mixture components in pure stand. At booting and milky ripe stages, plants sampled in the mixtures were separated according to the variety. No significant difference was found between the disease level in pure stand and in mixed stand either for the susceptible variety nor for the $P c h l$ one.
\end{abstract}

Triticum aestivum / Pseudocercosporella herpotrichoides / cultivars / variety mixture

Résumé - Le développement du piétin-verse sur une variété sensible, une variété possédant le gène de résistance $P c h 1$ et leur association à parts égales est étudié dans six situations qui diffèrent par le lieu, la protection fongicide ou le mode de contamination. Aucune différence de niveau de maladie n'est observée entre l'association et la moyenne des deux composants au stade deux nœuds. Aux stades gonflement et grain laiteux, les variétés sont identifiées dans l'association et notées séparément. Le niveau de maladie en culture pure n'est pas significativement différent de celui de l'association ni pour la variété sensible, ni pour la variété $P c h l$.

Triticum aestivum / Pseudocercosporella herpotrichoides / variété résistante / association variétale

\section{INTRODUCTION}

L'association variétale est une technique proposée par certains auteurs (de Vallavieille-Pope et al, 1988) pour réduire le développement des épidémies de certains parasites, en particulier ceux qui présentent des populations différenciées par leurs gènes de virulence spécifiques des résistances de l'hôte. Le principe est d'associer sur une même parcelle des variétés pourvues de résistances différentes : ainsi, une partie de la population de la plante cultivée demeure résistante aux parasites présents dans la population parasite locale et ralentit la propagation de celle-ci, aboutissant à une diminution du niveau final de maladie. Cette

\footnotetext{
* Correspondance et tirés à part.
} 
méthode de lutte par la culture d'associations variétales est une solution pour réduire les effets des agents pathogènes tels que les rouilles et l'oïdium des céréales, et justifiée dans les systèmes de culture à intrants réduits (Wolfe et Barrett, 1980).

Des résultats intéressants sont obtenus dans le cas de la septoriose causée par Septoria nodorum, maladie à développement aérien pour laquelle les relations hôte-parasite sont peu spécifiques et le niveau de résistance de l'hôte peu élevé (Jeger et al, 1981), ainsi que dans le cas d'Helminthosporium victoriae, champignon du sol parasitant les tiges d'avoine (Ayanru et Browning, 1977). Pour le piétin-verse des céréales causé par Pseudocercosporella herpotrichoides, aucun effet d'association sur le développement de la maladie n'est mis en évidence par de Vallavieille-Pope et al (1991) mais ces résultats sont obtenus avec des variétés toutes assez sensibles à la maladie.

Des variétés de blé tendre peu sensibles au piétin-verse ont été sélectionnées : elles possèdent un gène de résistance au stade plantule $P$ chl issu de l'espèce voisine Aegilops ventricosa qui se traduit par un ralentissement de la pénétration des gaines par le parasite (Doussinault et al, 1983). Dans l'état actuel de la sélection, le rendement des variétés disponibles n'atteint pas le niveau des meilleures variétés cultivées. Ces variétés pourraient être valorisées en association avec des variétés sensibles mais productrices, si elles permettaient une limitation significative de la maladie.

Dans ce travail, nous étudions le développement du piétin-verse dans des associations composées d'une variété possédant le gène $P c h l$ et d'une variété sensible.

\section{MATÉRIEL ET MÉTHODES}

Deux variétés de blé tendre d'hiver sont cultivées pures ou associées dans six situations devant représenter différents niveaux de développement du piétin-verse.
Chaque couple de variétés est constitué d'une variété sensible, soit Soissons ou Génial, et d'une variété ou lignée possédant le gène de résistance au piétin-verse Pchl (Pactole ou la lignée RE201 obtenue à la station d'amélioration des plantes Inra de Rennes-Le-Rheu) (tableau I). Le mélange des semences est réalisé de façon à ce que chaque génotype soit représenté par le même nombre de grains susceptibles de germer. La dose de semis est déterminée avec un objectif de levée de 300 plantes par $\mathrm{m}^{2}$. Les variétés sont identifiables dans les associations uniquement à partir du stade montaison.

Les essais sont implantés au champ en automne 1994 en trois lieux : Cintré (35), Pacé (35) et La Verrière (78). L'essai de Cintré comporte deux traitements, l'un contaminé artificiellement par le piétinverse, l'autre non. À La Verrière, trois traitements sont différenciés par leur niveau de protection fongicide : aucune protection spécifique contre le piétin-verse, traitement de semence au triticonazole $(120 \mathrm{~g}$ de $\mathrm{ma} / 100 \mathrm{~kg}$ de semences), traitement en végétation au cyprodinyl ( $750 \mathrm{~g}$ de ma/ha le 24/03). Les différentes combinaisons lieu, variétés, contamination et protection fongicide sont récapitulées dans le tableau I. Les dispositifs expérimentaux sont en blocs randomisés avec quatre répétitions à La Verrière, trois à Cintré et deux à Pacé. Pour l'analyse, l'effet du lieu n'est pas étudié mais chaque combinaison lieu-traitement constitue une situation, codée dans le tableau I.

La contamination artificielle est réalisée au stade 2-feuilles en épandant une culture de Pseudocercosporella herpotrichoides var herpotrichoides, obtenue sur milieu grains d'avoine, à raison de $10 \mathrm{~g} / \mathrm{m}^{2}$.

Des prélèvements de plantes sont réalisés à quatre stades de développement du blé : tallage (du 2/02 au $15 / 02$ ), début montaison (du 7/04 au 15/04), gonflement (du 15/05 au 18/05) et grain laiteux (du 15/06 au 20/06). L'échantillon est constitué en arrachant 5 à 8 plantes consécutives sur une ligne en 5 endroits de la parcelle pour les cultures pures et 10 à 15 plantes pour les associations. Les plantes présentant des symptômes de piétin-verse sont comptées et les résultats exprimés en fréquence de plantes atteintes. Aux deux derniers stades de notation, les variétés associées sont identifiées et observées séparément. Une note de 1 à 4 est attribuée à chacune en fonction de la surface de la section nécrosée par le piétin-verse (1: moins de $10 \%$ de la section nécrosée, $2: 10$ à $30 \%, 3: 30$ à $60 \%, 4:$ plus de $60 \%$ ). Les résultats sont exprimés en fréquence de

Tableau I. Variétés, modes de contamination et protections fongicides des différents essais.

\begin{tabular}{lccccc}
\hline $\begin{array}{l}\text { Lieux } \\
\text { expérimentaux }\end{array}$ & Code & $\begin{array}{c}\text { Variété } \\
\text { sensible }\end{array}$ & $\begin{array}{c}\text { Variété } \\
\text { résistante }\end{array}$ & Contamination & $\begin{array}{c}\text { Protection } \\
\text { fongicide }\end{array}$ \\
\hline Pacé & PA & Génial & RE201 & Non & Aucune \\
Cintré & CIC & Génial & RE201 & Oui & Aucune \\
La Verrière & CINC & Génial & RE201 & Non & Aucune \\
& LV1 & Soissons & Pactole & Non & Aucune \\
& LV2 & Soissons & Pactole & Non & Triticonazole \\
& LV3 & Soissons & Pactole & Non & Cyprodinil \\
\hline
\end{tabular}


tiges atteintes et indice de maladie (note moyenne pour l'ensemble des tiges observées).

L'interprétation statistique porte sur les fréquences, après transformation angulaire de Bliss. Au stade plantule, les résultats de l'association sont comparés aux résultats de la moyenne des deux parents par la méthode des contrastes. Au stade adulte nous disposons pour chaque variété de résultats correspondant aux deux types de culture : pure et association. Les différences sont appréciées par le test de F de Fisher.

Afin d'avoir des informations sur la structure des populations de $P$. herpotrichoides présentes dans chaque essai, des isolements sont réalisés à partir de tiges malades de la variété sensible, de façon à obtenir 20 isolats par lieu. Ceux-ci sont caractérisés par leur vitesse de croissance et leur résistance au triadiménol in vitro.

\section{RÉSULTATS}

\section{Type de souches}

Les souches à croissance dite normale (souches $\mathrm{N}$ ), par opposition aux souches à croissance lente (souches L) (Cavelier et al; 1987), sont majoritaires dans toutes les situations : $100 \%$ à La Verrière, $80 \%$ à Pacé, $90 \%$ à Cintré dans la partie contaminée et $60 \%$ dans la partie non contaminée. Tous les isolats de type $\mathrm{L}$ sont résistants au triadiménol in vitro. La proportion d'isolats de type $\mathrm{N}$ résistants au triadiménol est de $100 \%$ à $\mathrm{La}$ Verrière, $23 \%$ à Pacé et $12 \%$ à Cintré.

\section{Développement du piétin-verse au stade plantule}

Au stade tallage, le piétin-verse n'est visible qu'à Cintré dans la parcelle contaminée artificiellement. La fréquence de plantes attaquées est $93 \%$ pour la variété sensible Génial, $40 \%$ pour RE201 et $51 \%$ pour l'association, c'est-à-dire un peu moins que la moyenne des deux composants.

À La Verrière, la pulvérisation fongicide de cyprodinil est appliquée juste avant la notation début montaison : nous n'avons donc différencié que deux situations à ce stade sur ce site. Une fréquence élevée de plantes présentant des symptômes est observée à La Verrière et dans le traitement ayant reçu une contamination artificielle à Cintré. La comparaison entre le développement de la maladie à La Verrière dans la situation ayant reçu un traitement de semence au triticonazole (LV2) et le témoin non traité (LV1) montre que la molécule utilisée ne permet pas de diminuer le niveau du piétin-verse. Les fréquences de plantes attaquées dans les associations variétales sont très proches de la moyenne des deux composants ; l'étude des contrastes ne fait apparaître aucune différence significative à un seuil inférieur à $10 \%$ entre les résultats de l'association et sa valeur théorique (tableau II).

\section{Développement du piétin-verse au stade gonflement}

À ce stade, les situations sont bien différenciées. Le développement de la maladie est important dans la zone contaminée artificiellement à Cintré et dans celles n'ayant pas reçu de protection fongicide en végétation à La Verrière (LV1 et LV2). Les symptômes de piétin-verse sont faibles sur ce dernier site dans les parcelles protégées par le cyprodionil (LV3) ainsi qu'à Pacé (tableau III).

La différence de développement de la maladie entre variété sensible et résistante est importante dans toutes les situations.

Dans tous les cas, les fréquences de tiges atteintes et les indices de maladie sont proches

Tableau II. Pourcentages de plantes attaquées par le piétin-verse au début de la montaison pour deux variétés et leur association.

\begin{tabular}{lccccc}
\hline Situation & $\begin{array}{c}\text { Variété } \\
\text { sensible }\end{array}$ & $\begin{array}{c}\text { Variété } \\
\text { résistance }\end{array}$ & Mélange & $\begin{array}{c}\text { Moyenne des } \\
\text { deux variétés }\end{array}$ & $\mathrm{P}^{a}$ \\
\hline CIC & $96^{\mathrm{b}}$ & 14 & 51 & 55 & 0,4878 \\
CINC & 29 & 0 & 6 & 15 & 0,5296 \\
LV1 & 71 & 12 & 41 & 42 & 0,6869 \\
LV2 & 79 & 14 & 49 & 67 & 0,6665 \\
PA & 10 & 1 & 8 & 6,0999 \\
\hline
\end{tabular}

\footnotetext{
a Probabilité associée au test $\mathrm{t}$ pour le contraste entre la valeur en mélange et la valeur moyenne des deux variétés en culture pure ;
}

${ }^{\mathrm{b}}$ fréquence de tiges attaquées $(\%)$. 
Tableau III. Pourcentage de tiges attaquées et indice de maladie au stade gonflement dans six situations sur deux types de variétés en culture pure et en mélange.

\begin{tabular}{lccccccc}
\hline & \multicolumn{3}{c}{ Variété sensible } & & \multicolumn{3}{c}{ Variété résistance } \\
\cline { 2 - 3 } Situation & culture pure & mélange & $\mathrm{P}^{a}$ & & culture pure & mélange & $\mathrm{P}^{a}$ \\
\hline CIC & $77,1^{\mathrm{b}}$ & 88,3 & 0,2249 & & 33,2 & 26,7 & 0,4661 \\
& $2,3^{\mathrm{c}}$ & 2,6 & 0,3068 & & 0,5 & 0,4 & 0,6164 \\
CINC & 52,5 & 36,4 & 0,3893 & & 1,8 & 2,3 & 0,7728 \\
& 1,3 & 1,1 & 0,6239 & & 0,1 & 0,1 & 0,6090 \\
LV1 & 75,4 & 83,8 & 0,0709 & & 11,9 & 8,3 & 0,1214 \\
& 2,19 & 2,38 & 0,3845 & & 0,2 & 0,1 & 0,1840 \\
LV2 & 75,1 & 87,1 & 0,0453 & & 17,0 & 20,8 & 0,4827 \\
& 2,3 & 2,6 & 0,0594 & & 0,3 & 0,3 & 0,6517 \\
LV3 & 20,4 & 20,2 & 0,9916 & & 0,0 & 2,3 & 0,783 \\
& 0,4 & 0,4 & 0,6282 & & 0,0 & 0,0 & 0,3039 \\
PA & 21,3 & 20,5 & 0,9757 & & 4,3 & 1,3 & 0,8442 \\
& 0,3 & 0,5 & 0,7441 & & 0,0 & 0,0 & 0,6392 \\
\hline
\end{tabular}

a Probabilité associée au test $F$ de Fisher (pour les fréquences, le $F$ est calculé sur données transformées par la transformation angulaire de Bliss); ${ }^{b}$ fréquence de tiges attaquées $(\%) ;{ }^{c}$ indice moyen de maladie $(0$ à 4$)$.

aussi bien en culture pure que dans les associations variétales. Dans un seul cas, le test statistique conduit à accepter une différence significative au seuil de $5 \%$ (situation LV2 variété sensible) où la fréquence de tiges de la variété associée présentant des symptômes est plus élevée (87 \%) qu'en culture pure $(75 \%)$.

\section{Développement du piétin-verse au stade grain laiteux}

À ce stade, plus de $90 \%$ de tiges atteintes sont observées sur la variété sensible dans les situations où le niveau de maladie est le plus élevé. Les indices de maladie sont alors supérieurs à 3 . Les fréquences les plus faibles sont notées à Cintré dans la partie non contaminée $(56 \%)$ et à $\mathrm{La}$ Verrière dans le traitement ayant fait l'objet de la protection fongicide en végétation $(59,3 \%)$. La fréquence de tiges atteintes est élevée à Pacé sur la variété sensible $(70,4 \%)$, alors que le pourcentage de plantes malades début montaison était faible sur ce site $(10 \%)$.

Dans tous les cas, la variété résistante montre des fréquences de tiges atteintes moins importantes que celles de la variété sensible, même si celles-ci sont parfois assez élevées comme à Cintré dans la partie contaminée où elle atteint 70 $\%$. L'indice de maladie est cependant inférieur à 2 alors qu'il atteint 3,5 pour la variété sensible, ce qui indique que la gravité des symptômes est différente entre les deux types variétaux (tableau IV).
Il y a peu de différences entre les résultats obtenus en culture pure et associations variétales. Aucune différence n'est significative pour la variété sensible. Pour la variété résistante la probabilité associée au test $\mathrm{F}$ de Fisher est inférieure à 0,05 dans trois cas : pour l'indice de maladie (CIC et CINC) et pour la fréquence (LV3). Les différences observées restent cependant très faibles.

\section{DISCUSSION}

Dans les trois lieux d'expérimentation, les souches présentes sont presque exclusivement du type $\mathrm{N}$. Les suivis des populations de $P$ herpotrichoides dans des parcelles de blé dans la moitié nord de la France montrent que depuis quelques années les souches de type $\mathrm{L}$ sont prépondérantes dans de nombreux cas (Mingeon, 1994). Sachant que les deux types de souches ont des rythmes de développement différents, les conclusions de cette étude ne sont pas généralisables.

Le triticonazole appliqué en traitement des semences ne permet pas de diminuer la maladie, mais les essais de résistance in vitro montrent que les souches prélevées à La Verrière sont résistantes au triadiménol. La résistance aux triazoles est aujourd'hui très fréquente dans les populations de $P$ herpotrichoides et s'applique à toutes les molécules de cette famille qui ont été étudiées (Leroux et Gredt, 1985). La résistance des souches au triticonazole, molécule appartenant comme le triadi- 
Tableau IV. Pourcentage de tiges attaquées et indice de maladie au stade grain laiteux dans six situations sur deux types de variétés en culture pure et en mélange.

\begin{tabular}{|c|c|c|c|c|c|c|}
\hline \multirow[b]{2}{*}{ Situation } & \multicolumn{3}{|c|}{ Variété sensible } & \multicolumn{3}{|c|}{ Variété résistance } \\
\hline & culture pure & mélange & $\mathrm{P}^{\prime \prime}$ & culture pure & mélange & $\mathrm{P}^{a}$ \\
\hline \multirow[t]{2}{*}{$\mathrm{ClC}$} & $87,9^{b}$ & 92,1 & 0,0986 & 67,0 & 70,4 & 0,1454 \\
\hline & $3,4^{\circ}$ & 3,5 & 0,4073 & 1,9 & 1,7 & 0,0186 \\
\hline \multirow[t]{2}{*}{ CINC } & 56,0 & 53,3 & 0,8298 & 22,7 & 11,3 & 0,0898 \\
\hline & 1,7 & 1,5 & 0,6152 & 0,4 & 0,2 & 0,0499 \\
\hline \multirow[t]{2}{*}{ LVI } & 91,7 & 89,8 & 0,8208 & 35,3 & 45,7 & 0,3566 \\
\hline & 3,4 & 3,3 & 0,7443 & 0,2 & 0,1 & 0,3545 \\
\hline \multirow[t]{2}{*}{ LV2 } & 91,8 & 92,5 & 0,6125 & 53,7 & 53,3 & 0,9679 \\
\hline & 3,5 & 3,5 & 0,8326 & 1,3 & 2,3 & 0,9043 \\
\hline \multirow[t]{2}{*}{ LV3 } & 59,3 & 63,9 & 0,6502 & 17,6 & 23,1 & 0,0365 \\
\hline & 1,7 & 1,8 & 0,6895 & 0,3 & 0,5 & 0,3863 \\
\hline \multirow[t]{2}{*}{ PA } & 70,4 & 59,5 & 0,3966 & 37,1 & 35,7 & 0,9477 \\
\hline & 2,3 & 1,9 & 0,1967 & 0,9 & 1,0 & 0,9608 \\
\hline
\end{tabular}

a Probabilité associée au test $F$ de Fisher (pour les fréquences, le $F$ est calculé sur données transformées par la transformation angulaire de Bliss) ; ${ }^{\mathrm{b}}$ fréquence de tiges attaquées (\%); "indice moyen de maladie ( 0 à 4$)$.

ménol à la famille des triazoles, est l'explication la plus probable de l'absence d'efficacité de cette molécule. Dans cette hypothèse, nos résultats confirment que la résistance observée in vitro se traduit bien par une perte d'efficacité de la molécule in vivo.

Le développement du piétin-verse est inférieur, sur les deux variétés possédant le gène $P c h l$ que nous avons utilisées, à celui observé sur les variétés sensibles. La différence est encore visible en fin de végétation, même si ces variétés ne sont pas totalement résistantes. Certains génotypes possédant ce gène de résistance au stade plantule ne possèdent pas une résistance très élevée au stade adulte et, en cas de forte attaque, les symptômes sur tige peuvent être aussi importants que sur certaines variétés sensibles. Le bon comportement de Pactole et RE201 dans les conditions sévères de développement de la maladie, telles que nous les avons obtenues à Cintré ou à La Verrière, pourrait refléter chez ces génotypes une faible sensibilité au stade adulte.

Comme pour de Vallavieille-Pope et al (1991), et malgré l'utilisation de variétés présentant un bon niveau de résistance, nous n'avons pas mis en évidence d'effet de la culture en mélange sur le développement du piétin-verse. Deux effets sont invoqués pour expliquer l'effet du mélange de génotypes sur le niveau de maladie : l'éloignement entre les plantes sensibles et l'effet de barrière. De nombreux travaux ont montré que le niveau d'attaque par le piétin-verse diminue avec la densité de plantes (Glynne et Slope, 1959 ; Mielke,
1970 ; Huet, 1986). Une des explications de ces phénomènes est la diminution de la probabilité de rencontre entre l'inoculum et la plante (Burdon et Chilvers, 1982). On aurait pu penser que cet effet se retrouverait dans la culture en mélange ; or, dans nos essais pour lesquels l'association variétale correspond à une diminution de $50 \%$ de la densité de plantes sensibles, nous observons le même développement de la maladie sur la variété sensible qu'en culture pure. On ne peut a fortiori parler d'effet barrière. La majorité des études théoriques ou expérimentales qui ont montré l'efficacité des mélanges ont été faites sur des maladies à cycles multiples. Dans ce cas, même si l'effet est faible sur une génération de l'agent pathogène, il peut devenir sensible en fin d'épidémie (Wolfe, 1985). Chez $P$ herpotrichoides, le temps de latence est relativement long et on a longtemps considéré que seule l'infection primaire avait de l'importance dans le développement de la maladie. Des travaux, basés sur des suivis de sporulation (Moreau et al, 1990), ou sur la modélisation des épidémies (Colbach et Meynard, 1995), ont montré l'importance des cycles secondaires et tertiaires pour expliquer les niveaux de maladie observés en fin de végétation. Dans une des situations que nous avons suivies (Pacé), la fréquence de plantes présentant des symptômes à la sortie de l'hiver est faible, et la fréquence de tiges contaminées au stade grain laiteux atteignait $70 \%$ sur la variété sensible. C'est dans ce type de développement prolongé dans le temps de la maladie que les cycles secondaires sont considérés comme importants mais, même dans ce cas, nous n'observons 
pas l'effet de protection de l'association variétale contre le développement de la maladie sur la variété sensible.

Il ressort de ces résultats que l'utilisation de la variabilité génétique des variétés au sein du champ cultivé ne permet pas de rapprocher le niveau de développement du piétin-verse sur la variété sensible dans l'association variétale, de celui observé sur la variété la plus résistante. Dans le cas de cette maladie, l'intérêt d'associer des variétés au lieu de les cultiver séparément ne peut être révélé qu'en situation de très forte attaque parasitaire, si la variété résistante peut compenser les pertes liées à la variété sensible.

\section{RÉFÉRENCES}

Ayanru DKG, Browning JA (1977) Effect of heterogenous oat populations on the epiphytotic development of Victoria blight. New Phytol 79, 613-623

Burdon JJ, Chilvers GA (1982) Host density as a factor in plant disease ecology. Annu Rev Phytopathol 20, 143-166

Cavelier N, Rousseau M, Lepage D (1987) Variabilité de Pseudocercosporella herpotrichoides agent $\mathrm{du}$ piétin-verse des céréales : comportement in vivo de deux types d'isolats et d'une population en mélange. Z Pflanzenkr Pflanznschutz 94, 591-599

Colbach N, Meynard JM (1995) Soil tillage and eyespot: influence of crop residues distribution on disease developement and infection cycle. Eur J Plant Patho 101, 601-611

Doussinault G, Delibes A, Sanchez-Monge R, GarciaOlmedo F (1983) Transfer of a dominant gene for resistance to eyespot disease from a wild grass to hexaploid wheat. Nature 303, 698-700

Glynne MD, Slope DB (1959) Effect of previous wheat crops, seed rate and nitrogen on eyespot, take-all, weeds and yields of two varieties of winter wheat. Ann Appl Biol 47, 187-199

Huet P (1986) Influence du système de culture sur le piétin-verse du blé. In : Les rotations céréalières intensives. Dix années d'études concertées InraOnic-ITCF 1973-1983, Inra Paris, 95-111

Jeger MJ, Jones DJ, Griffiths E (1981) Disease progress of non specialised fungal pathogens in intraspecific mixed stands of cereal cultivars. II. Field experiments. Ann Appl Biol 98, 199-210

Leroux P, Gredt M (1985) Caractéristiques des souches de Pseudocercosporella herpotrichoides, agent du piétin-verse des céréales, résistantes à des inhibiteurs de la biosynthèse des stérols. C R Acad Sci Paris sér III 301, 785-788

Mielke H (1970) Befallstoleranz und Halmbruchresistenz verschiedener Weizensorten gegen Cercosporella herpotrichoides Fron. Z Pflanzenzïcht 64, 248-288

Migeon JL, Mathon MP, Chudzicki AM, Leroy JP (1994) Piétin-verse des céréales : cartographie 1993. Phytoma 458, 24-26

Moreau JM, Van Schingen JC, Maraite H (1990) Épidémiologie de Pseudocercosporella herpotrichoides var acuformis et var herpotrichoides sur froment d'hiver, démonstration d'un cycle secondaire Med Fac Landbouww Rijksuniv Gent 55, 889-898

de Vallavieille-Pope C, Goyeau H, Pinard F, Vergnet C, Mille B (1988) Integrating varietal mixtures and fongicide treatments: preliminary studies of a strategy for controlling yellow rust of wheat. In: Integrated Crop Protection in Cereals Proc Meeting EC Experts Group, Littlehampton, UK, 25-27 november 1986, 199-207

de Vallavieille-Pope C, Goyeau H, Lannou C, Mille B (1991) Pour lutter contre les maladies foliaires, la culture de variétés en mélange. Phytoma 424, 28-36

Wolfe MS, Barrett JA (1980) Can we lead the pathogen astray? Plant Disease 64, 148-155

Wolfe MS (1985) The current status and prospects of multiline cultivars and variety mixtures for disease resistance. Annu Rev Phytopathol 23, 251-273 